\title{
Dead cone due to parton virtuality
}

\author{
Trambak Bhattacharyya* and Jan-e Alam \\ Theoretical Physics Division, Variable Energy Cyclotron Centre, 1/AF, Bidhannagar, Kolkata-700064
}

(Dated: December 10, 2018)

\begin{abstract}
A general expression for the dead cone of gluons radiated by virtual partons has been derived. The conventional dead cone for massive on-shell quarks and the dead cone for the massless virtual partons have been obtained by using different limits of the general expression. Radiative suppression due to the virtuality of initial parton jets in Heavy-Ion Collisions (HIC) has been discussed. It is observed that the suppression caused by the high virtuality is overwhelmingly large as compared to that on account of conventional dead-cone of heavy quarks. The dead cone due to virtuality may play a crucial role in explaining the observed similar suppression patterns of light and heavy quarks jets in heavy ion collisions at Relativistic Heavy Ion Collider (RHIC).
\end{abstract}

PACS numbers: $12.38 . \mathrm{Mh}, 24.85 .+\mathrm{p}, 25.75 . \mathrm{Nq}$

Energy loss of high energy partons in Quark Gluon Plasma (QGP) which is expected to be produced in nuclear collisions at Large Hadron Collider (LHC) and RHIC energies has drawn huge attention recently. The interaction of energetic partons with QGP is reflected through the measured suppression $\left(R_{\mathrm{A} A}\right)$ of high transverse momentum $\left(p_{T}\right)$ hadrons. The two dominant mechanisms which cause this suppression are: (i) elastic collision of the propagating partons with the quarks, antiquarks and gluons in the thermal bath created in the $\mathrm{HIC}$ and (ii)radiation of gluons by the propagating partons due to its interactions with the QGP.

Classically the induced radiation takes place due to the jiggling of the propagating particle in the medium. Since the heavier particles jiggle less, induced energy loss is expected to be smaller [dead cone effect 1, 2] (see also [3, 4])] for HQ compared to that for light particles. However, the experimental data from RHIC indicates similar energy loss by heavy quarks [ $[, 6]$ and light partons [7, 8] in the measured kinematic range. Various reasons like the anomalous mass dependence of the radiative process due to the finite size of the QGP [9], development of dead cone due to high virtuality of the partons resulting from the dismantling of colour fields during the initial hard collisions [10] have been proposed as the possible reasons for this observation. The authors in 11] concluded that the reduction in the energy loss of HQ due to radiative process is attributed to the dead cone effect. It is fair to state that at this moment the issue of the observed similar suppression of heavy and light hadrons is yet to be settled. However, the comparable magnitude of suppression for light and heavy flavoured hadrons indicates that a common mechanism may be at work for the radiative processes.

The dead cone is a conical domain around the direction of motion of the massive partons within which the radiation of gluon is prohibited. The mass of the quark decides the size of the dead cone. However, the high en-

\footnotetext{
*trambakb@vecc.gov.in
}

ergy quarks and gluons produced from the hard collisions of the partons from the colliding nucleons are off-shell and their colour fields are stripped off, i.e. they have no field to radiate. Therefore, the partons' virtuality creates its own dead-cone which may be large depending on the magnitude of the virtuality. The fobiden zone around the direction of motion of the partons due to its virtuality will be called virtual dead cone here. The conventional dead cone (due to the mass of the quark) becomes important when the virtuality of the quarks reduces to zero. The objective of the present work is to propose a quantitative estimate of the dead cone due to virtuality of quark jets and to investigate its interplay with the conventional dead cone 2] (see also [12]).

The dead cone suppression obtained in [2] actually has an analogy with radiated power distribution of a nonrelativistic, accelerating charge particle. The average power radiated per unit solid angle is given by [13]:

$$
\frac{d P}{d \Omega} \propto|\dot{\vec{\beta}}|^{2} \sin ^{2} \theta
$$

where $\theta$ is the angle between acceleration $\dot{\vec{\beta}}$ of the particle and the direction of propagation of radiation, $\vec{n}$. This is a simple $\sin ^{2} \theta$ behavior showing no radiation at $\theta=0$ (or $\mathrm{n} \pi, \mathrm{n} \in \mathrm{Z}$ ). It will be shown later that the behavior of Eq. 1 is, indeed, similar to what one gets for conventional dead cone 2].

We can take up the process $e^{+} e^{-} \longrightarrow Q \bar{Q} g$, (where $\mathrm{Q}$ denotes heavy quark) to show that the soft part of the radiative amplitude, when squared, gives rise to the dead-cone suppression factor of heavy quarks. We will follow the same line of arguments as that of Ref. [14] for virtual heavy quarks to give a formula for the suppression factor due to virtuality of quark jets. It will be argued that for non-zero virtuality the suppression factor is independent of the current mass of quark. The absence of virtuality in heavy quarks results in a behavior similar to that obtained in [2]; and last but not the least, we will also verify the absence of 'conventional' dead-cone suppression for on-shell light quarks from the same formula.

The tree-level Feynman diagrams for gluon radiation by heavy quarks/anti-quarks in the process $e^{+} e^{-} \longrightarrow$ 
$Q \bar{Q} g$ are depicted in Fig. 1 .

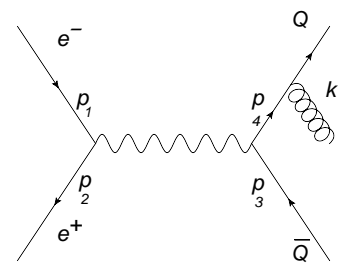

(a)

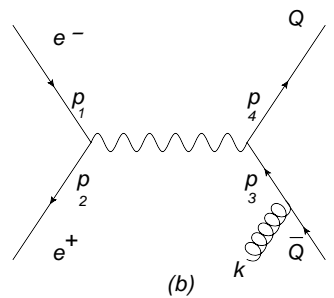

(b)
The first term of Eq. 5 which is proportional to $p_{4} \cdot \epsilon^{a^{*}}(k)$ can be defined as the soft part of amplitude [14]. For dominance of the soft part of $\mathcal{M}_{a}$ we need, $\left|\overrightarrow{p_{i}}\right| \sin \theta>>\omega$, where $\theta$ is the angle between parent (off-shell) quark and the gluon emitted with energy, $\omega$. Using similar arguments for Fig. 1 (b), we get the total soft amplitude given by:

$$
\left[\mathcal{M}_{s o f t}\right]_{a+b} \sim 2\left(\frac{p_{4} \cdot \epsilon^{a^{*}}(k)}{V^{2}+2 p_{4} \cdot k}-\frac{p_{3} \cdot \epsilon^{a^{*}}(k)}{V^{2}+2 p_{3} \cdot k}\right)
$$

FIG. 1: Tree-level Feynman diagram for gluon radiation by (a) quark and (b) anti-quark.

The amplitude for the process shown in Fig. 1a can be written as:

$$
\begin{aligned}
\mathcal{M}_{a}= & \frac{g_{s} e_{Q} e^{2}}{s}\left(t^{a}\right)_{j}^{i}\left[\bar{v}\left(p_{2}\right) \gamma_{\mu} u\left(p_{1}\right)\right] \\
& \times\left[\bar{u}_{i}\left(p_{4}\right) \xi^{a *}(k) \frac{\left(p_{4}+\not k+m_{Q}\right)}{\left(\left(p_{4}+k\right)^{2}-m_{Q}^{2}\right)} \gamma^{\mu} v^{j}\left(p_{3}\right)\right]
\end{aligned}
$$

where $e_{Q}$ is the quark electric charge, e is electric charge, $a, i, j$ are the color indices and $s=\left(p_{1}+p_{2}\right)^{2}$. We assume that $p_{3}^{2}=p_{4}^{2} \neq m_{Q}^{2}$. For off-shell particles the virtuality can be defined through the parameter $V$ as:

$$
V^{2}=q^{2}-m_{Q}^{2}
$$

where $q^{2}$ is four-momentum square of external virtual particles, $q^{2}=m_{Q}^{2}$ implies $V=0$, i.e. the particle becomes on-shell. We build our calculation on very small value of $\mathrm{V}$ so that the of-shell external quarks are at the vicinity of being on-shell. Under such approximations we can use Dirac's equation for quark and anti-quark.

The denominator of Eq. 2 can be expanded in terms of $V$ to get,

$$
\begin{aligned}
\left(p_{4}+k\right)^{2}-m_{Q}^{2} & =p_{4}^{2}+2 p_{4} \cdot k+k^{2}-m_{Q}^{2} \\
& =V^{2}+2 p_{4} \cdot k
\end{aligned}
$$

with $k^{2}=0$ for on-shell radiated gluon. The numerator of Eq. 2 can be simplified by using the anti-commutator, $\left\{p_{i}, p_{f}\right\}=2 p_{i} \cdot p_{j}$ and Dirac equation $\bar{u}(p)(\not p-m)=0$ as follows:

$$
\begin{aligned}
\bar{u}_{i}\left(p_{4}\right) \xi^{a^{*}}(k)\left(p_{4}+\not k+m_{Q}\right)= & \bar{u}_{i}\left(p_{4}\right) \xi^{a^{*}}(k)\left(\not p_{4}+m_{Q}\right) \\
& +\bar{u}_{i}\left(p_{4}\right) \epsilon^{a^{*}}(k) \not k \\
= & \bar{u}_{i}\left(p_{4}\right)\left(-\not p_{4}+m_{Q}\right) \xi^{a^{*}}(k) \\
& +2 p_{4} \cdot \epsilon^{a^{*}}(k) \bar{u}_{i}\left(p_{4}\right) \\
& +\bar{u}_{i}\left(p_{4}\right) \xi^{a^{*}}(k) \not k \\
= & 2 p_{4} \cdot \epsilon^{a *}(k) \bar{u}_{i}\left(p_{4}\right) \\
& +\bar{u}_{i}\left(p_{4}\right) \xi^{a^{*}}(k) \not k
\end{aligned}
$$

This part of the amplitude originates from the gluon radiation. We call it the 'radiation factor', $R$. To obtain the cross-section of the process we square the amplitude and sum over the relevant colour and spin degrees of freedom. Algebraic manipulation of $R$ gives rise to the conventional dead-cone suppression of on-shell massive quarks. Now, with our assumption of low virtuality of heavy quarks, $\mathrm{R}$ is, again, expected to lead us to a quantitative understanding of the suppression due to offshellness. Its interplay with the suppression due to quark mass for on-shell quarks can be obtained simultaneously, too.

The radiation factor $\mathrm{R}$, when squared and summed over spin, gives

$$
\begin{aligned}
\sum_{\text {spin }}|R|^{2}= & \left(\frac{p_{4}^{\alpha}}{V^{2}+2 p_{4} \cdot k}-\frac{p_{3}^{\alpha}}{V^{2}+2 p_{3} . k}\right) \\
& \left(\frac{p_{4}^{\beta}}{V^{2}+2 p_{4} \cdot k}-\frac{p_{3}^{\beta}}{V^{2}+2 p_{3} \cdot k}\right) \\
& \times \sum \epsilon_{\alpha}^{a^{*}}(k) \epsilon_{\beta}^{a^{\prime}}(k) \\
= & \left(\frac{p_{4}^{\alpha}}{V^{2}+2 p_{4} \cdot k}-\frac{p_{3}^{\alpha}}{V^{2}+2 p_{3} \cdot k}\right) \\
& \left(\frac{p_{4}^{\beta}}{V^{2}+2 p_{4} \cdot k}-\frac{p_{3}^{\beta}}{V^{2}+2 p_{3} \cdot k}\right) \\
& \times\left(-\eta_{\alpha \beta}(k) \delta^{a a^{\prime}}\right) \\
= & -\left|\frac{p_{4}}{V^{2}+2 p_{4} \cdot k}-\frac{p_{3}}{V^{2}+2 p_{3} \cdot k}\right|^{2} \\
= & \frac{2 p_{4} \cdot p_{3}}{\left(V^{2}+2 p_{4} \cdot k\right)\left(V^{2}+2 p_{3} \cdot k\right)} \\
& -\frac{p_{4}^{2}}{\left(V^{2}+2 p_{4} \cdot k\right)^{2}} \\
= & 2 R_{43}-R_{44}^{2}-R_{33}
\end{aligned}
$$

Our problem now boils down to simplify the quantity, $2 R_{43}-R_{44}-R_{33}$ to get the necessary suppression factor.

To proceed further we take the following form of the four-momenta for the radiating and the radiated parti- 
cles:

$$
\begin{aligned}
p_{4} & =E_{4}\left(1, \overrightarrow{\beta_{4}}\right) \\
p_{3} & =E_{3}\left(1, \overrightarrow{\beta_{3}}\right) \\
k & =\omega(1, \vec{n})
\end{aligned}
$$

where $\beta_{i}$ is the velocity of the quark carrying a momentum $p_{i}$ and energy $E_{i}$ and the gluon is emitted along direction $\vec{n}$ with energy $\omega$. If the radiating quark and antiquark were on-shell then one could write, for quark, say,

$$
\begin{aligned}
& p_{4}^{2}=m_{Q}^{2}=E_{4}^{2}\left(1-\beta_{4}^{2}\right) \\
& \Rightarrow\left(1-\beta_{4}^{2}\right)=\frac{m_{Q}^{2}}{E_{4}^{2}}=\frac{1}{\gamma_{4}^{2}}
\end{aligned}
$$

where $\gamma$ is the Lorentz factor. But, for our present purpose we define,

$$
\left(1-\beta_{4}^{2}\right)=\frac{q^{2}}{E_{4}^{2}}=\frac{1}{\gamma_{4}^{2}}
$$

which is almost but not exactly the Lorentz factor as we have considered off-shellness of the partons here. The factor $\omega^{2} R_{43}$ can be written as:

$$
\begin{aligned}
& \omega^{2} R_{43}= \\
& \frac{\omega^{2} E_{3} E_{4}\left(1-\beta_{3} \beta_{4} \cos \theta_{34}\right)}{\left(V^{2}+2 E_{4} \omega\left(1-\beta_{4} \cos \theta_{4}\right)\right)\left(V^{2}+2 E_{3} \omega\left(1-\beta_{3} \cos \theta_{3}\right)\right)}
\end{aligned}
$$

In the equal- $\beta$ frame, where both particles have equal velocities back to back such that $\beta_{3}=\beta_{4} ; \theta \equiv \theta_{3}=$ $\theta_{4}-\pi$; and $\theta_{34}=\theta_{4}-\theta_{3}=\pi$, we have $E_{3}=E_{4}=E$. In this frame we can write,

$$
\begin{aligned}
& \omega^{2} R_{43}= \\
& \frac{\left(1+\beta^{2}\right)}{\left(\frac{V^{2}}{\omega E}+2(1-\beta \cos \theta)\right)\left(\frac{V^{2}}{\omega E}+2(1+\beta \cos \theta)\right)}
\end{aligned}
$$

Similarly,

$$
\omega^{2} R_{33}=\frac{\left(1-\beta^{2}\right)}{\left(\frac{V^{2}}{\omega E}+2(1+\beta \cos \theta)\right)^{2}}
$$

and

$$
\omega^{2} R_{44}=\frac{\left(1-\beta^{2}\right)}{\left(\frac{V^{2}}{\omega E}+2(1-\beta \cos \theta)\right)^{2}}
$$

Combining Eqs. 12,13 and 14 we get the spectrum:

$$
\begin{aligned}
F= & \omega^{2}\left(2 R_{43}-R_{44}-R_{33}\right) \\
& =4 \beta^{2}\left(\frac{\frac{V^{4}}{\omega^{2} E^{2}}+\frac{4 V^{2}}{\omega E}+4 \sin ^{2} \theta}{\left(\frac{V^{4}}{\omega^{2} E^{2}}+\frac{4 V^{2}}{\omega E}+4\left(1-\beta^{2} \cos ^{2} \theta\right)\right)^{2}}\right)
\end{aligned}
$$

Next we explore different limits of $F$ given in Eq. 15, (i) For zero virtuality $(V=0)$ of the massive quark, Eq. 15 reduces to the conventional dead cone factor:

$$
F=\omega^{2}\left(2 R_{43}-R_{44}-R_{33}\right) \longrightarrow \frac{\beta^{2} \sin ^{2} \theta}{\left(1-\beta^{2} \cos ^{2} \theta\right)^{2}}
$$

This is the well-known conventional dead cone for a gluon emitted by a massive quark. The divergence of the factor is shielded by the quark mass through $\beta(<1)$.

(ii) Now we investigate the light quark limit $(\beta=1)$. For $V=0, \beta=1$,

$$
F \sim \frac{1}{\sin ^{2} \theta}
$$

For light quarks Eq. 17 ensures the absence of dead-cone suppression at $\theta=0, \pi$ when virtuality is small.
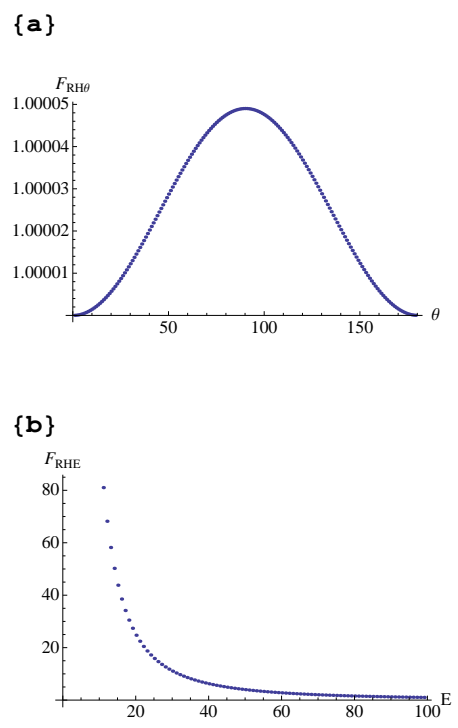

FIG. 2: (Colour online) The variation of $(\mathrm{a}) F_{\mathrm{R} H \theta}(E, \theta)$ with $\theta$ for $E=1 \mathrm{GeV}$, (b) $F_{\mathrm{R} H E}(E, \theta)$ with $E$ for $\theta=45 \mathrm{deg}$ for heavy quarks $(\beta<1)$.

For quantitative variation of $F$ with energy $E$ of the radiating partons, and angle $\theta$ between the radiating and 
radiated partons, we replace virtuality of heavy quark by its energy E. The emitted gluon carry a fraction of parent parton energy.

In Fig. 2 we display the dead cone factor, $F$ for heavy quarks. The variation of $F_{\mathrm{R} H \theta}=F(E=$ $1 G e V, \theta) / F(E=1 G e V, \theta=0)$ with $\theta$ is depicted in Fig. 2(a) for heavy quarks $(\beta<1)$. It is interesting to note that for small $E$ and consequently for small virtuality the suppression is similar to that of a conventional dead cone for massive quarks. In Fig. 2 (b) the variation of $F_{\mathrm{R} H E}=F(E, \theta=\pi / 4) / F(E=100 \mathrm{GeV}, \theta=\pi / 4)$ with $E$ is displayed for heavy quarks.

In Fig. 3 the dead cone factor for light quarks is illustrated. In Fig. 3 (a) the variation of $F_{\mathrm{R} L \theta}=F(E=$ $1 G e V, \theta) / F(E=1 G e V, \theta=0)$ with $\theta$ is shown for light partons $(\beta=1)$. It is important to note that the variation of $F_{\mathrm{R} L \theta}$ with $\theta$ for light quark with low virtuality is drastically different from $F_{\mathrm{RH} \theta}$ for heavy quark. This is obvious because for low virtuality the light partons are not subjected to any dead cone suppression at $\theta=0$ and $\pi$ unlike heavy quarks. Moreover, the $\sin ^{-2} \theta$ behaviour for light quarks (Eq. 17) ensures a minimum at $\theta=\pi / 2$ as opposed to a maximum at the same $\theta$ for heavy quarks. In Fig. 3 (b) the variation of $F_{\mathrm{R} L E}=F(E, \theta=\pi / 4) / F(E=100 \mathrm{GeV}, \theta=\pi / 4)$ with $E$ is depicted. We note that the suppression is large for high $E$ (virtuality), similar to heavy quarks, which indicates that the dead cone effects because of large virtuality overwhelms the dead cone due to the mass of the quarks.

In Figs. 4 (a) and (b) we plot $F_{\mathrm{R} L \theta}$ and $F_{\mathrm{R} H \theta}$ as a function of $\theta$ for $E=20 \mathrm{GeV}$. We observe that the radiative suppression due to virtuality is of the same magnitude for both the light (Fig. 4 a) and heavy quarks (Fig. 4 b). This leads to the conclusion that suppression due to virtuality exists irrespective of the current quark mass i.e. off-shell quarks, heavy or light are always exposed to similar radiative suppression. It is apparent from the results displayed in Figs. 4(a) and 4(b) that the suppression factor given by Eq 15 may be responsible for the observed similar suppression patterns for light [7, 8] and heavy [5, 6] hadrons of RHIC data.

In summary, we have derived an expression for the dead cone of gluons radiated by virtual partons. The conventional dead cone for massive on-shell quarks and the dead cone for the virtual partons have been obtained as different limits of the general expression. We have demonstrated the interplay between dead-cone radiative suppression of heavy quarks and that due to virtuality of quark jets. For on-shell light quarks, the absence of radiative suppression at $\theta=0, \pi$ is ascertained too. We observe that the radiative suppression due to virtuality of initial parton jets in HIC may be overwhelmingly large as compared to that due to conventional dead-cone of heavy quarks. Therefore, the dead cone caused by virtuality may play a crucial role in explaining the observed similar suppression patterns of light and heavy quark jets in heavy ion collisions at RHIC.

Acknowledgment: Fruitful discussions with Suras-

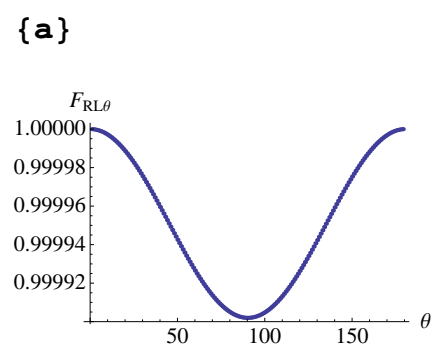

$\{\mathbf{b}\}$

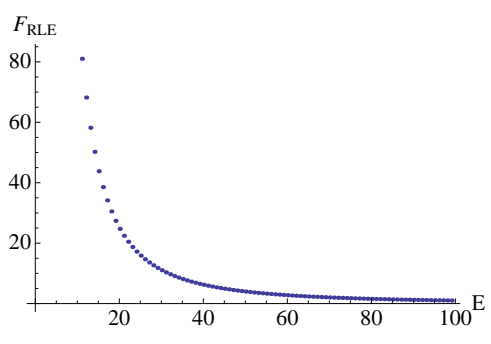

FIG. 3: (Colour online) The variation of (a) $F_{\mathrm{R} L \theta}(E, \theta)$ with $\theta$ for $E=1 \mathrm{GeV}$, (b) $F_{\mathrm{R} L E}(E, \theta)$ with $E$ for $\theta=\pi / 4$ for light partons $(\beta=1)$.

ree Mazumder, Sourav Sarkar, Santosh K. Das and P. Tribedy are acknowledged. TB is supported by the Department of Atomic Energy, Govt of India.
[1] R. K. Ellis, W. J. Stirling and B. R. Webber, QCD and Collider Physics, Cambridge University Press, Cambridge, 1996.

[2] Y. L. Dokshitzer and D. E. Kharzeev, Phys. Lett. B 519,
$199(2001)$

[3] B. W. Zhang, E. Wang and X.-N. Wang, Phys. Rev. Lett. 93, 072301 (2004).

[4] N. Armesto, A. Dainese, C. A. Salgado and U. A. Wiede- 

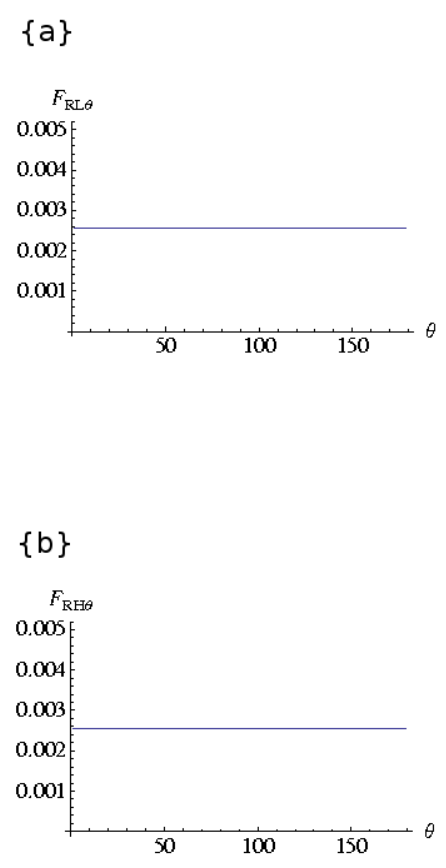

mann, Phys. Rev. D 71, 054027 (2005)

[5] B. I. Abelev et al. (STAR Collaboration), Phys. Rev. Lett. 98, 192301 (2007).

[6] A. Adare et al. (PHENIX Collaboration), Phys. Rev. Lett. 98, 172301 (2007).

[7] B. I. Abelev et al. (STAR Collaboration), Phys. Lett. B 655, 104 (2007).

[8] S. S. Adler et al., (PHENIX Collaboration), Phys. Rev. Lett. 96, 202301 (2006).

[9] B. G Zakharov, JETP Lett. 86, 444(2007); P. Aurenche and B. G. Zakharov, JETP Lett. 90, 237 (2009).

[10] B. Z. Kopeliovich, I. K. Potashnikova, I. Schmidt, Phys. Rev. C 82, 037901(2010)

[11] Roy A. Lacey et al., Phys. Rev. Lett.103,142302(2009)

[12] R. Abir, C. Greiner, M. Martinez, M. G. Mustafa and J. Uphoff, Phys. Rev. D 85, 054012 (2012).

[13] J.D. Jackson, Classical Electrdynamics, John Wiley and Sons Pvt Ltd, Third Edition

[14] K. M. Paul Ph. D. Thesis, University of Illinois at Urbana-Champaign, 2002
FIG. 4: The variation of normalized $F$ with $\theta$ for $E=20 \mathrm{GeV}$, (a) light partons, (b)massive quarks. The $F$ is normalized by its value at $E=20 \mathrm{GeV}$ and $\theta=0$. 